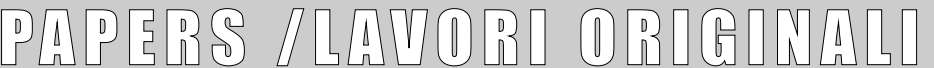

\section{Indagine epidemiologica locale dell'eziologia delle infezioni delle vie urinarie (IVU) nosocomiali e comunitarie e dell'antibiotico-sensibilità degli uropatogeni.}

\author{
Agostina Ronca', Sabrina Brenci', Luisa Santoriello', Anna Marchese ${ }^{2}$, Simona Roveta $^{2}$ \\ 'Struttura Semplice Dipartimentale di Microbiologia - Azienda Ospedaliera Santa Corona, Via XXV Aprile 38, I7027 \\ Pietra Ligure (SV). \\ 2Università di Genova, DISCAT - Sezione di Microbiologia, Largo R. Benzi 10, 16I32 Genova.
}

Key words: Urinary tract infections, Escherichia coli, uropathogens, antibiotic resistance.

Local surveillance study on etiology of community - and hospital-acquired urinary tract infections (UTI) and antimicrobial susceptibility of uropathogens.

\section{SUMMARY}

Background: Urinary tract infections (UTIs) are common infectious diseases that can be associated with substantial morbidity. During the last decade, resistance to ampicillin and co-trimoxazole has increased in Escherichia coli, the most common uropathogen, and recent reports have shown increasing resistance even to fluoroquinolones. The aim of this local surveillance study was to determine the distribution of bacterial strains isolated from outpatients and inpatients with UTIs and antibiotic susceptibility patterns to antimicrobial agents currently used in the treatment of pathogens causing these infections.

Materials and methods: Between January and March 2006 a total of 1596 urine specimens, 968 from outpatients and 628 from inpatients, respectively, were recovered. Urinary pathogens isolated were 235, identification and antimicrobial susceptibility testing were performed by Vitek II. The following antimicrobial agents were tested: ampicillin, amoxicillin-clavulanic acid, ceftazidime, imipenem, co-trimoxazole, ciprofloxacin, gentamicin and nitrofurantoin. $E$ test ${ }^{\circledR}$ method were used to study the production of extended spectrum beta-lactamases (ESBL). Results: The most frequent pathogen found was Escherichia coli (68.5\%), followed by Klebsiella spp. (8.5\%), Proteus mirabilis (7.6\%), and Enterococcus spp. (6\%). E. coli resistance rates less than 10\% was observed for ceftazidime, imipenem and nitrofurantoin. In strains isolated from outpatients resistance to ampicillin and trimethoprim-sulfamethoxazole was $37 \%$ and $19 \%$, respectively, and resistance to fluoroquinolones was about $20 \%$. Resistance rates of $E$. coli was significantly higher in complicated nosocomial-acquired infection: ampicillin $53.6 \%$, cotrimossazole $35.7 \%$ and ciprofloxacin 33.9\%. ESBL producer strains were 7 E.coli (4.3\%) and 6 Proteus spp. (33\%).

Conclusions: This study confirmed that E. coli and other Enterobacteriaceae are the predominant bacterial pathogens envolved in UTIs. Currently, the empirical use of co-trimoxazole and ampicillin is not recommended. Because of the increase in fluoroquinolones resistance among uropathogens patients who can benefit from these antimicrobial agents must be selected. Third-generation cephalosporins still have high sensitivity rates (although the the emergence of extended-spectrum beta-lactamase-producing enterobacterial should be taken into account). Urine culture and antimicrobial susceptibility testing is the "gold standard" for diagnosis of UTI and selection of appropriate treatment.

\section{INTRODUZIONE}

Le infezioni delle vie urinarie (IVU), pur essendo associate a una letalità piuttosto bassa, rappresentano a causa dell'elevata frequenza e morbilità un rilevante problema clinico. Vi è consenso sul fatto che, in assenza di fattori di rischio concomitanti, quali per esempio l'età avanzata e il diabete, il microrganismo più frequentemente in causa sia rappresentato da Escherichia coli, con incidenze che oscillano, nei diversi studi e nelle differenti situazioni geografiche, dal $70 \%$ al $90 \%(1,2,7$, $10,14,21,23)$. Questo dato è stato recentemente confermato anche in Italia da differenti studi che hanno evidenziato, per le IVU gestite a livello ambulatoriale, una netta prevalenza di E. coli (5, 6, 22). L'eziologia delle IVU non complicate comunitarie è inoltre frequentemente attribuibile ad altri Gram-negativi, come Proteus spp., Klebsiella spp. e Enterobacter spp., più raramente a Gram-positivi come Enterococcus spp. e Staphylococcus spp. $(12,14)$. L'eziologia delle IVU nosocomiali, che sono pressoché tutte complicate, differisce notevolmente da quella delle infezioni comunitarie. L'importanza relativa di $E$. 
coli viene ridimensionata sino a incidere per meno del $50 \%$ dei casi studiati, mentre assumono maggior rilievo altre specie batteriche (sia Grampositive che Gram-negative) che possono essere acquisite dal paziente dalla popolazione microbica ospedaliera $(3,7,11,17,18,26-28)$.

Negli ultimi anni si è riscontrato, seppure con delle differenze tra i vari Paesi, un costante incremento dei livelli di resistenza nei confronti degli antibiotici più frequentemente utilizzati nella terapia delle IVU. Il fenomeno riguarda principalmente farmaci come cotrimossazolo e ampicillina, tuttavia, sono state osservate in alcuni aree geografiche anche incidenze piuttosto elevate di resistenza ai fluorochinoloni $(4,8,9,11,13-16$, 19, 20, 24-28).

Questo studio si propone di indagare in un determinato periodo di tempo (un trimestre) l'eziologia sia delle IVU comunitarie che di quelle nosocomiali nella nostra realtà locale e in seguito di analizzare lo spettro di sensibilità agli antibiotici dei principali patogeni coinvolti.

\section{MATERIALI E METODI}

Nel periodo gennaio-marzo 2006, presso la Struttura Semplice Dipartimentale di Microbiologia dell'Azienda Ospedaliera Santa Corona, sono stati analizzati 1596 campioni di urina, 968 dei quali provenienti da pazienti comunitari e 628 di origine nosocomiale. Sono stati esclusi dall'indagine campioni consecutivi provenienti dallo stesso paziente nonché prelievi da catetere o da sacchetto.

La carica batterica dei campioni è stata valutata con il Sistema automatizzato Uro-Quick, quelli risultati positivi allo screening sono stati seminati sia su terreno arricchito (Columbia agar sangue $5 \%$ ), per permettere la crescita di microrganismi Gram-positivi e Gram-negativi, sia su terreno selettivo (Mac-Conkey agar) per favorire l'isolamento dei soli Gram-negativi. Le piastre sono state quindi incubate in aerobiosi a $37^{\circ} \mathrm{C}$ per $18-24$ ore. Sono stati inclusi nello studio solamente campioni monomicrobici aventi una carica batterica superiore a $10^{5} \mathrm{CFU} / \mathrm{ml}$. Per l'identificazione dei germi e l'esecuzione dell'antibiogramma è stato impiegato il metodo automatizzato VitekII (bioMérieux Italia S.p.A).

È stata infine valutata la sensibilità di $E$. coli, il più comune patogeno delle vie urinarie, e delle altre Enterobacteriaceae più frequentemente coinvolte nelle IVU, Klebsiella spp. e Proteus spp., nei confronti delle principali classi di farmaci: penicilline (ampicillina, AMP), penicilline protette (amoxicillina-acido clavulanico, AMC), cefalosporine di terza generazione (ceftazidime, CAZ), carbapenemici (imipenem, IPM), fluorochinoloni (ciprofloxacina, CIP), cotrimossazolo (SXT), aminoglicosidi (gentamicina, GN) e nitrofurantoina (FT). I suddetti microrganismi sono, inoltre, stati sottoposti al saggio per la presenza di ß-lattamasi a spettro esteso (ESBL) tramite E-test (B) (Biolife Italiana Srl, Milano) (cefotaxime/ceftazidime).

\section{RISULTATI}

I campioni d'urina risultati positivi sono stati 235 (pari al 14.7\%), 138 dei quali comunitari e 97 nosocomiali (14.2 e $15.4 \%$ rispettivamente).

In totale sono stati isolati $161 \mathrm{E}$. coli, 20 Klebsiella spp. (15 K. pneumoniae e 5 K. oxytoca), 18 Proteus mirabilis, 14 Enterococcus spp., altri 14 stipiti Gram negativi (4 Pseudomonas aeruginosa, 4 Citrobacter freundii, 3 Morganella morganii, 3 Enterobacter aerogenes) e 8 Gram positivi (4 Streptococcus agalactiae, 3 Staphylococcus epidermidis, 1 Staphylococcus aureus). Nella Figura I sono riportate le percentuali di isolamento dei vari microrganismi relativamente al totale dei campioni positivi; i batteri Gram negativi si confermano, anche secondo questo studio, come principali responsabili delle infezioni delle vie urinarie $(90.6 \%)$. L'agente eziologico più diffuso risulta essere 1'E. coli (68.5\%), seguito dalle altre Enterobacteriaceae: Klebsiella spp (8.5\%) e $P$. mirabilis (7.6\%). I Gram positivi, invece, incidono per meno del $10 \%$ e tra questi il genere più diffuso è Enterococcus spp (6\%).

La Figura II evidenzia la distribuzione dei patogeni in relazione alla provenienza nosocomiale o comunitaria: nei campioni comunitari E. coli predomina rispetto a quelli nosocomiali in rapporto di 2 a 1 . Per quanto riguarda il genere Klebsiella, la provenienza del campione non ha inciso sul ritrovamento del batterio, mentre per $P$. mirabilis ed Enterococcus spp. la frequenza di isolamento è risultata molto più alta nei campioni provenienti dai pazienti nosocomiali rispetto a quelli comunitari.

Tra gli E. coli isolati da pazienti ospedalizzati solo il $34 \%$ si è dimostrato completamente sensibile a tutti gli antibiotici saggiati, mentre tra $\mathrm{i}$ comunitari questa percentuale raggiungeva quasi il $52 \%$.

Sempre nell'ambito di questo patogeno, considerando i campioni di origine nosocomiale, le maggiori incidenze di resistenza sono state riscontrate nei confronti di AMP (53.6\%) seguita da SXT 
(35.7\%), CIP (33.9\%) e GN (16.1\%). Percentuali di resistenza inferiori al $10 \%$ sono state osservate solo per CAZ (8.9\%), FT (3.6\%) e IPM (0\%). Per quanto riguarda gli stipiti di E. coli di origine comunitaria, il $37.1 \%$ presentava resistenza a AMP, il $21.9 \%$ a CIP e il $19.0 \%$ a SXT. Percentuali di resistenza inferiori al $10 \%$ sono state riscontrate per GN (9.5\%), AMC (6.7\%), FT (4.8\%), CAZ (1.9\%) e IPM (0\%) (Figura III).

Tutti gli isolati del genere Klebsiella si sono dimostrati resistenti alle penicilline ed il $60 \%$ di quelli provenienti da campioni nosocomiali anche a FT, questa percentuale scendeva al $40 \%$ negli stipiti comunitari. Solamente 2 ceppi $(10 \%)$, uno nosocomiale e l'altro comunitario, sono risultati resistenti ai fluorochinoloni. Nessun isolato è risultato resistente a CAZ, IPM o GN.

Il $61.5 \%$ dei $P$. mirabilis di provenienza nosocomiale mostrava resistenza alle penicilline, il $53.8 \%$ a SXT, il $46.2 \%$ a CIP e GN, il $23.1 \%$ a CAZ, AMC e IPM. Lo spettro di sensibilità dei ceppi comunitari non differiva sostanzialmente da quello degli stipiti nosocomiali.

Sono risultati produttori di ß-lattamasi a spettro esteso 7 stipiti di E. coli $(4.3 \%)$ che presentavano resistenza alle cefalosporine di terza generazione (cefotaxime e ceftazidime), 5 provenienti da pazienti ospedalizzati e 2 di origine comunitaria. Tra gli isolati appartenenti al genere Klebsiella non sono stati individuati stipiti produttori di ESBL. Sei ceppi di P. mirabilis (33\%), 4 nosocomiali e 2 comunitari veicolavano ESBL (Figura IV). È stata svolta un'indagine sulla storia clinica dei pazienti da cui provenivano i 2 E. coli e i $2 P$. mirabilis comunitari ESBL positivi; due soggetti, uno portatore di $E$. coli e l'altro di P. mirabilis, erano stati ospedalizzati in precedenza e durante il ricovero le loro urinocolture erano risultate positive per P. mirabilis ESBL produttore.

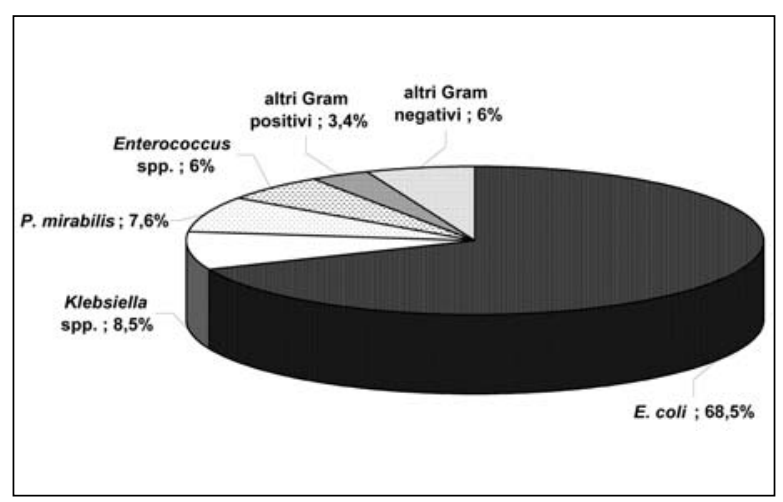

Figura I. Percentuali di isolamento dei vari microrganismi relativamente ai 235 campioni positivi.

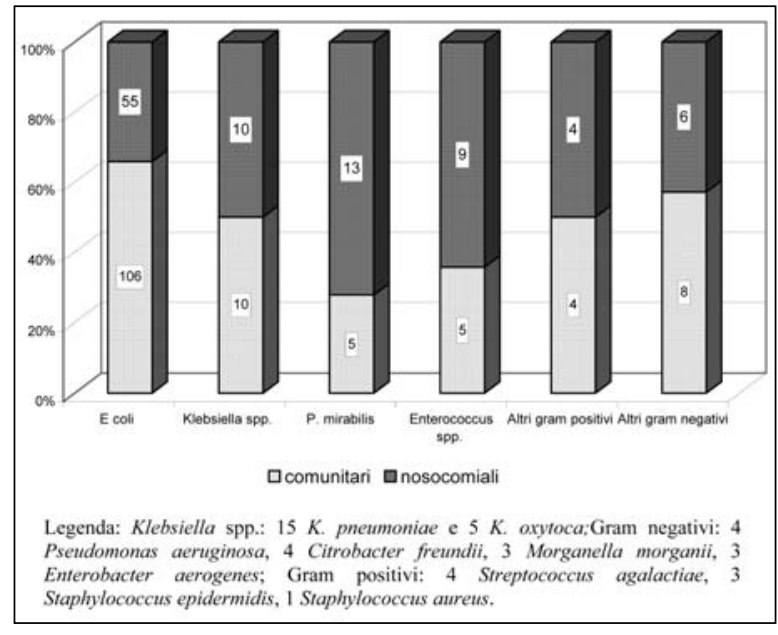

Figura II. Distribuzione dei microrganismi (e numero di ceppi isolati) sulla base della provenienza nosocomiale o comunitaria.

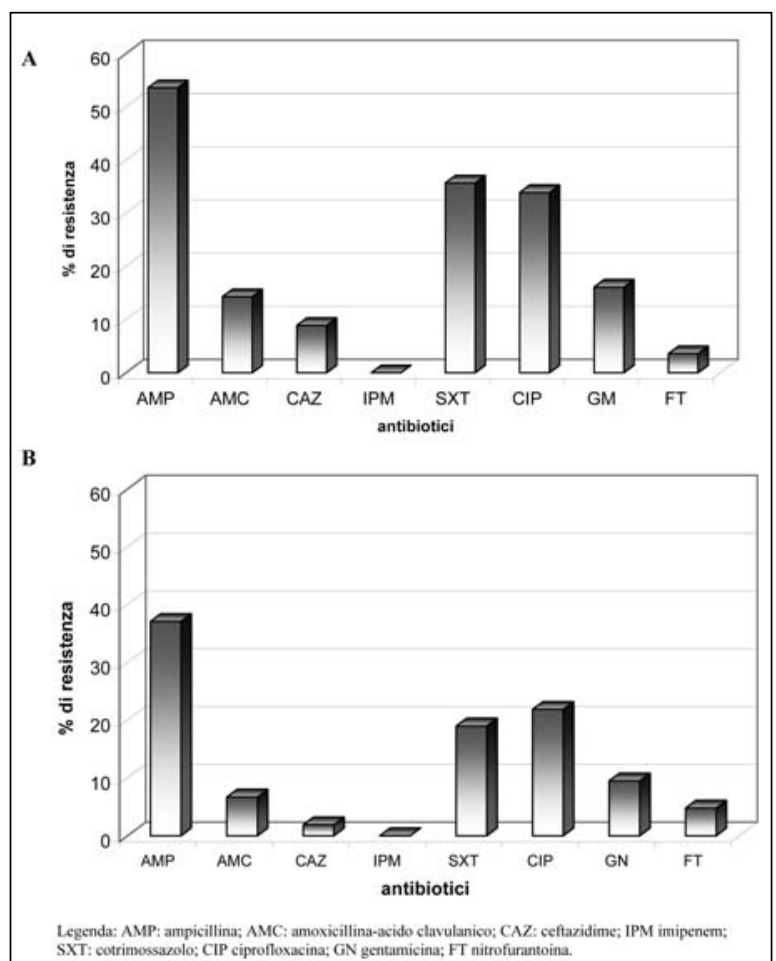

Figura III. Percentuali di resistenza alle principali classi di farmaci nei 55 ceppi di E: coli nosocomiali (A) e nei 106 stipiti comunitari (B).

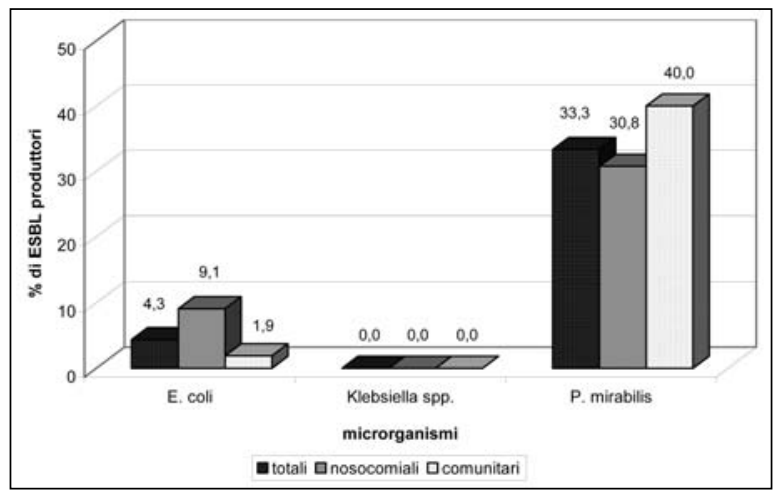

Figura IV. Diffusione di ESBL nelle Enterobacteriaceae 


\section{CONCLUSIONI}

Analogamente a quanto riportato in letteratura, le Enterobacteriaceae dominano nell'eziologia delle IVU anche nella nostra realtà locale. In questo studio è stata evidenziata una presenza significativa di E. coli (quasi il 70\%) nei campioni d'urina positivi, con una percentuale più elevata in quelli provenienti da pazienti comunitari. Per quanto riguarda la sensibilità di questo patogeno nei confronti delle principali classi di farmaci impiegati in terapia, percentuali di diffusione della resistenza superiori al $20 \%$ sono state evidenziate nei confronti di ampicillina, cotrimossazolo e fluorochinoloni. Solamente ceftazidime, imipenem e nitrofurantoina hanno mostrato percentuali di sensibilità in vitro maggiori del $90 \%$. Dal momento che l'isolamento di ceppi dotati di resistenze multiple non rappresenta un evento raro, la terapia delle infezioni urinarie (soprattutto di quelle acquisite in ambito nosocomiale) non può essere affrontata con un approccio puramente empirico. Vista la disponibilità di tecnologie di accertamento microbiologico semplici, standardizzate e predittive del successo terapeutico (l'uso improprio di molecole inattive in vitro può facilmente condurre a fallimenti) il modo più razionale per affrontare i quadri clinici più impegnativi consiste nell'adozione di una terapia mirata. Nei suddetti casi, pertanto, appare opportuno prelevare un campione urinario prima dell'inizio del trattamento antibiotico per procedere all'isolamento del patogeno, alla determinazione dello spettro di sensibilità e all'instaurazione della terapia più appropriata. L'ottimizzazione delle strategie di antibioticoterapia riveste grande importanza nelle IVU, sia per evitare la diffusione di ceppi particolarmente resistenti, sia perché queste infezioni incidono in modo rilevante oltre che sulla qualità di vita dei pazienti anche sui costi sanitari correlati alla loro gestione.

\section{BIBLIOGRAFIA}

1. Alos JI. Epidemiology and etiology of urinary tract infections in the community. Antimicrobial susceptibility of the main pathogens and clinical significance of resistance. Enferm Infecc Microbiol Clin. 2005; 23 Suppl 4: 3-8.

2. Andreu A, Alos JI, Gobernado M, Marco F, De La Rosa M, Garcia-Rodriguez JA. Grupo Cooperativo Espanol para el Estudio de la Sensibilidad Antimicrobiana de los Patogenos Urinarios. Etiology and antimicrobial susceptibility among uropathogens causing community-acquired lower urinary tract infections: a nationwide surveillance study. Enferm Infecc Microbiol Clin. 2005; 23: 4-9.
3. Bagshaw SM, Laupland KB. Epidemiology of intensive care unit-acquired urinary tract infections.Curr Opin Infect Dis. 2006; 19: 67-71.

4. Barisic Z, Borzic E, Kraljevic KS, Carev M, Zoranic V, Kaliterna V. Rise in ciprofloxacin resistance in Escherichia coli from urinary tract infections from 1999-2004. Int J Antimicrob Agents. 2005; 25: 550-551.

5. Bosisio V, Stella R, Castagna S, Marchetti F. A prospective descriptive study on the practice of management of urinary tract infections by general pratictioners in Italy: epidemiology (Part I). Archivio Italiano di Urologia e Andrologia 2003; 75: 1-5.

6. Fadda G, Nicoletti G, Schito GC, Tempera G. Antimicrobial susceptibility patterns of contemporary pathogens from uncomplicated urinary tract infections isolated in a multicenter Italian survey: possible impact on guidelines. J Chemother. 2005; 17: 251-7.

7. Franco AV. Recurrent urinary tract infections. Best Pract Res Clin Obstet Gynaecol. 2005; 19: 861-73.

8. Goettsch W, van Pelt W, Nagelkerke N, et al. Increasing resistance to fluoroquinolones in Escherichia coli from urinary tract infections in the netherlands. J Antimicrob Chemother. 2000; 46: 223-228.

9. Gordon KA, Jones RN, SENTRY Participant Groups (Europe, Latin America, North America). Susceptibility patterns of orally administered antimicrobials among urinary tract infection pathogens from hospitalized patient in North America: comparison report to Europe and Latin America. Results from the SENTRY Antimicrobial Surveillance Program (2000). Diagn Microbiol Infect Dis. 2003; 45: 295 301.

10. Grude N, Tveten Y, Jenkins A, Kristiansen BE. Uncomplicated urinary tract infections. Bacterial findings and efficacy of empirical antibacterial treatment. Scand J Prim Health Care. 2005; 23: 115-9.

11. Johansen TE, Cek M, Naber KG, Stratchounski L, Svendsen MV, Tenke P; PEP and PEAP-study investigators; Board of the European Society of Infections in Urology. Hospital acquired urinary tract infections in urology departments: pathogens, susceptibility and use of antibiotics. Data from the PEP and PEAP-studies. Int J Antimicrob Agents. 2006; 28 Suppl 1: S91107.

12. Jones RN. Global epidemiology of antimicrobial resistance among community-acquired and nosocomial pathogens: a five-year summary from the SENTRY Antimicrobial Surveillance Program (1997-2001). Semin Respir Crit Care Med. 2003; 24: 121-134.

13. Junquera S, Loza E, Baquero F. Changes in the antimicrobial susceptibility of Escherichia coli isolates from nosocomial versus community-acquired urinary tract infections. Enferm Infecc Microbiol Clin. 2005; 23: 197-201.

14. Kahlmeter G. An international survey of the antimicrobial susceptibility of pathogens from uncomplicated urinary tract infections: the ECO-SENS Project. J Antimicrob Chenother. 2003; 51: 69-76.

15. Karaca Y, Coplu N, Gozalan A, Oncul O, Citil BE, Esen B. Co-trimoxazole and quinolone resistance in Escherichia coli isolated from urinary tract infections over the last 10 years. Int J Antimicrob Agents. 2005; 26:75-77.

16. Karlowsky JA, Hoban DJ, Decorby MR, Laing NM, Zhanel GG. Fluoroquinolone-resistant urinary isolates of Escherichia coli from outpatients are frequently multidrug resistant: results from the North American Urinary Tract Infection Collaborative AllianceQuinolone Resistance study. Antimicrob Agents 
Chemother. 2006; 50: 2251-2254.

17. Lau SM, Peng MY, Chang FY. Resistance rates to commonly used antimicrobial among pathogens of both bacteremic and non-bacteremic communityacquired urinary tract infection. J Microbiol Immunol Infect. 2004; 37: 185-191.

18. Laupland KB, Bagshaw SM, Gregson DB, Kirkpatrick AW, Ross T, Church DL. Intensive care unit-acquired urinary tract infections in a regional critical care system. Crit Care. 2005; 9: 60-5.

19. Lemort ML, Neuville S, Medus M, et al. Comparative susceptibility evolution in Escherichia coli from urinary tract infections in outpatients and inpatients at Perpignan hospital in 2002 and 2004. Pathol Biol (Paris). 2006, in stampa.

20. McDonald LC, Chen FJ, Lo HJ, et al. Emergence of reduced susceptibility and resistance to fluoroquinolones in Escherichia coli in Taiwan and contributions of distinct selective pressures. Antimicrob Agents Chemother. 2001; 45: 3084-3091.

21. Ronald A. The etiology of urinary tract infection: traditional and emerging pathogens. Am J Med. 2002; 113 (Suppl 1): S14-S19.

22. Speciale AM, Nicoletti G, Torrisi C, et al. Prevalenza e antibiotico-resistenza dei principali patogeni urinari responsabili di cistiti non complicate nella donna: lo studio ICEA 2. GIMMOC. 2004; 8 (Q1): 1-16.

23. Stratchounski LS, Rafalski VV. Antimicrobial susceptibility of pathogens isolated from adult patients with uncomplicated community-acquired urinary tract infections in the Russian Federation: two multicentre studies, UTIAP-1 and UTIAP-2. Int J Antimicrob Agents. 2006; 28 Suppl 1: S4-9.

24. Talon D, Lallemand-De-Conto S, Thouverez M, Bertrand X. E. coli: resistance to quinolones and betalactams of clinical strains isolated in the FrancheComte region of France. Pathol Biol (Paris). 2004; 52: 76-81.

25. Wagenlehner FM, Niemetz A, Dalhoff A, Naber KG. Spectrum and antibiotic resistance of uropathogens from hospitalized patients with urinary tract infections: 1994-2000. Int J Antimicrob Agents. 2002; 19: 557-564.

26. Wagenlehner FME, Naber KG. Emergence of antibiotic resistance and prudent use of antibiotic therapy in nosocomially acquired urinary tract infections. International Journal of Antimicrobial Agents 2004; 23 Suppl 1: S24-S29.

27. Wagenlehner FME, Naber KG. Treatment of bacterial urinary tract infections: presence and future. European Urology, 2006; 49: 235-244.

28. Wagenlehner FME, Weidnerb W, Naber KG. Emergence of antibiotic resistance amongst hospitalacquired urinary tract infections and pharmacokinetic/pharmacodynamic considerations. Journal of Hospital Infection 2005; 60: 191-200.

\section{Agostina Ronca \\ Struttura Semplice Dipartimentale di Microbiologia Azienda Ospedaliera Santa Corona. Via XXV Aprile 38, 17027 Pietra Ligure (SV) Tel.: 0196234377 - Fax: 0196234374 luisa.santoriello@ospedalesantacorona.it}

